\title{
The effect of acute morphine on obstructive sleep apnoea: a randomised double-blind placebo- controlled crossover trial
}

\author{
Luke Rowsell, ${ }^{1}$ Keith K H Wong, ${ }^{1,2}$ Brendon J Yee, ${ }^{1,2}$ Danny J Eckert, ${ }^{3}$ \\ Andrew A Somogyi, ${ }^{4}$ James Duffin, ${ }^{5}$ Ronald R Grunstein, ${ }^{1,2}$ David Wang ${ }^{1,2}$
}

\begin{abstract}
- Additional material is published online only. To view please visit the journal online (http://dx.doi.org/10.1136/ thoraxjnl-2018-211675).

${ }^{1}$ NHMRC Centre for Sleep and Chronobiology (CIRUS) and NHMRC CRE NeuroSleep, Woolcock Institute of Medical Research, Sydney Medical School, The University of Sydney, Glebe, New South Wales, Australia

${ }^{2}$ Department of Respiratory and Sleep Medicine, Royal Prince Alfred Hospital, Camperdown, New South Wales, Australia ${ }^{3}$ Neuroscience Research Australia (NeuRA) and the School of Medical Sciences, University of New South Wales, Randwick, New South Wales, Australia

${ }^{4}$ Discipline of Pharmacology, Adelaide Medical School, The University of Adelaide, Adelaide, South Australia, Australia ${ }^{5}$ Departments of Physiology and Anaesthesia, University of Toronto, Toronto, Ontario, Canada
\end{abstract}

\section{Correspondence to}

Dr David Wang, Department of Respiratory and Sleep Medicine, Royal Prince Alfred Hospital, Camperdown, NSW 2050, Australia;

david.wang@sydney.edu.au

Received 11 February 2018 Revised 26 June 2018 Accepted 23 July 2018 Published Online First 30 August 2018

\begin{abstract}
Objective Anaesthesiology guidelines suggest that opioids worsen obstructive sleep apnoea (OSA) despite no randomised controlled trial evidence. We therefore conducted a randomised controlled trial to evaluate the effects of a common clinical dose of morphine on OSA, and to identify clinical phenotype and genotype vulnerability to opioid-respiratory depression.
\end{abstract}

Methods Under a double-blind, randomised, crossover design, 60 male patients with OSA attended two visits to the hospital sleep laboratory, at least 1 week apart. Either $40 \mathrm{mg}$ controlled-release oral morphine or placebo was administered. Awake ventilatory chemoreflex tests were performed post dose and prior to overnight polysomnography monitoring. Blood was sampled before sleep and the next morning for toxicology and genotype analyses. Sleep time with oxygen saturation $\left(\mathrm{SpO}_{2}\right)$ $<90 \%$ (T90) was the primary outcome.

Results Despite a large inter-individual variability, $40 \mathrm{mg}$ morphine did not worsen T90 and apnoeahypopnoea index, and only decreased the $\mathrm{SpO}$, nadir by $1.3 \%$. In patients with severe OSA, a lower baseline $\mathrm{CO}_{2}$ ventilatory response threshold correlated with the worsening of T90, apnoea-hypopnoea index and oxygen desaturation index with morphine use. Patients with OSA and the A118G OPRM 1 polymorphism of $A / A$ and $A / G$ had a significantly different morphine effect on awake ventilatory chemosensitivity and T90 during sleep.

Conclusions $40 \mathrm{mg}$ oral controlled-release morphine did not worsen OSA in men, challenging traditional thinking that OSA will be worsened by opioids. Individual opioid response in patients with OSA may relate to baseline $\mathrm{CO}_{2}$ response threshold and OPRM1 genotype. Our study findings may pave the way for a precision medicine approach to avoid opioid-related risks.

Trial registration number The Australian and New Zealand Clinical Trial Registry, ACTRN12613000858796.

\section{INTRODUCTION}

Deaths related to prescription opioids have quadrupled in the past 15 years and account for approximately $90 \%$ of non-illicit drug poisoning deaths in the United States. ${ }^{1-3}$ Death from opioids is nearly always due to respiratory arrest, and often occurs during sleep when breathing is primarily regulated by automatic neurochemical control. ${ }^{4}$ Almost 50\% of middle-aged men and $25 \%$ of women have moderate to severe obstructive sleep apnoea (OSA). ${ }^{5}$ OSA is often unrecognised and undiagnosed. ${ }^{6}$

\section{Key messages}

What is the key question?

- What is the effect of a common clinical dose of acute oral morphine on obstructive sleep apnoea (OSA) and who is vulnerable to morphine-induced respiratory depression?

What is the bottom line?

- We found that $40 \mathrm{mg}$ oral controlled-release morphine did not worsen OSA overall, but there is a large inter-individual variability which may relate to individual chemoreflex and genotype.

Why read on?

- As the first major randomised controlled trial in this field, our study findings challenge previous pain medicine and anaesthesiology guidelines that suggest that opioids systematically worsen OSA severity, and the clinical phenotype and genotype findings may pave the way for a precision medicine approach to identify those most vulnerable to opioid-related harm.

Perioperative care of individuals with OSA is a major concern due to the potential risk of harm. ${ }^{78}$ Indeed, while randomised controlled trial (RCT) evidence is lacking, OSA is considered a risk factor for postoperative complications as highlighted in recent guidelines and reviews. ${ }^{89}$ Therefore opioids are relatively contraindicated in pain management guidelines for people with OSA. ${ }^{10-12}$ The American Society of Anaesthesiologists ${ }^{7}$ and the Society of Anaesthesia and Sleep Medicine ${ }^{8}$ have issued practice guidelines for the perioperative management of patients with OSA to reduce the risk of adverse outcomes. However, the relevant recommendations were primarily based on retrospective case reports and consensus opinion. Consequently, there is a paucity of evidence concerning the effect of opioids on OSA to guide clinicians treating patients with chronic pain owing to the competing risks of inadequate treatment of pain and potentially fatal opioid overdose. To date, only one small controlled OSA study has been performed, comparing the effect of remifentanil $(n=10)$ with saline infusion $(n=9)$ in patients with OSA. ${ }^{13}$ Remifentanil infusion increased central sleep apnoea events and decreased obstructive events with no overall change 
in apnoea-hypopnoea index (AHI), and with marked inter-individual variability. ${ }^{13}$ The small sample size, lack of crossover design and use of an intravenous infusion to deliver the shortacting drug therefore limits the ability to draw robust conclusions about the standard oral opioid use at doses used to treat acute pain.

The pathophysiology of OSA is complex and the effects of opioids on OSA are not well understood. There may be large inter-individual variability in the effects of opioids, with OSA worsening in some and improving in others, which may be explained, at least in part, by inter-individual differences in OSA pathophysiology. ${ }^{13}{ }^{14}$ In our pilot study that included 10 people with mild to moderate OSA, $30 \mathrm{mg}$ of acute oral morphine paradoxically improved nocturnal oxygenation in those with the highest plasma morphine concentration, which was correlated with changes in awake ventilatory chemoreflexes. ${ }^{14}$ These findings led us to the hypothesis that certain ventilatory chemoreflex phenotypes may predict which patients with OSA are prone to respiratory depression, or conversely, experience reductions in OSA severity with morphine. Furthermore, polymorphisms in genes controlling opioid pharmacokinetics and/or pharmacodynamics may also influence the response to these drugs. ${ }^{15} \mathrm{Up}$ to $30 \%$ of variability in respiratory depression due to opioids is thought to be due to genetic factors. ${ }^{16}$

To address the evidence gap relating to opioid effects in patients with OSA, we conducted a randomised trial to evaluate the effect of a common clinical dose of acute oral morphine on OSA, and to identify patients with OSA who are vulnerable to morphine-induced respiratory depression by using clinical phenotyping and genotyping techniques. We hypothesised that morphine would have a beneficial effect on OSA in people with certain phenotypes/genotypes via a reduction in ventilatory chemosensitivity, and worsen OSA in others who have certain phenotypes/genotypes, rendering these patients at increased risk of respiratory arrest.

\section{METHODS}

\section{Study design and participants}

The MorphOSA trial was a randomised, double-blind, placebo-controlled crossover trial conducted at the sleep laboratory of the Royal Price Alfred Hospital, a tertiary teaching hospital of the University of Sydney. Sixty-eight untreated men with OSA were evaluated with 60 completed. Patients were recruited following diagnosis of OSA (AHI $\geq 5$ events/hour) from the sleep clinics at the Woolcock Institute of Medical Research or Royal Prince Alfred Hospital. Patients were included when they had a body mass index (BMI) $\leq 40 \mathrm{~kg} / \mathrm{m}^{2}$, awake peripheral oxygen saturation $\left(\mathrm{SpO}_{2}\right)>90 \%$ and nocturnal $\mathrm{SpO}_{2}$ nadir $\geq 60 \%$.

Patients were excluded if they had a severe medical comorbidity, history of drug abuse or allergy to morphine, were taking central nervous system active drugs such as sedative hypnotics and antipsychotics, a creatinine clearance (Cockroft-Gault) $<60 \mathrm{~mL} /$ min; abnormal lung function test, or a known history of $\mathrm{CO}_{2}$ retention ( $\mathrm{PCO}_{2}>45 \mathrm{mmHg}$ during wakefulness). Patients with other significant sleep disorders and concurrent continuous positive airway pressure (CPAP) users were also excluded.

All patients provided written informed consent, and the local ethics committee (NSW Sydney Local Health District SSWAHS trial X13-0045) approved the study.

\section{Randomisation and masking}

A computer programme produced two sequences with randomised permuted blocks with a block size of four. Based on screening stratification, patients were assigned using one of the two produced randomisation sequences to receive either morphine first, then placebo, or the reverse. Following screening, each patient was assigned a unique number in sequential, ascending, chronological order which corresponded to the treatment allocation and stratification. Morphine sulfate or identical placebo was prepared in opaque capsules by a hospital pharmacist who was not involved in patient assessments. Study investigators were blinded to treatment allocation for the duration of the study.

\section{Procedures}

On each study visit, $40 \mathrm{mg}$ controlled-release morphine (MS Contin one $30 \mathrm{mg}$ and one $10 \mathrm{mg}$ capsule) or placebo was administered at 17:30 following an evening meal. After administration, patients remained in the sleep laboratory and abstained from food or caffeine. Between 21:00 and 21:45, ventilatory chemoreflexes and $5 \mathrm{~min}$ ventilation were measured while awake, rested and seated. At 21:30 and 07:00 the next morning, blood samples $(5 \mathrm{~mL})$ were collected for plasma morphine and metabolite analysis, and opioid genotyping.

Overnight in-laboratory polysomnography was performed at around 22:00. Polysomnographic data were recorded and analysed using Respironics Alice-5 hardware and G3 software (Respironics, Murrysville, Pennsylvania, USA). Sleep and respiratory scoring was performed by a single, blinded senior poloysomnographic technologist using standard AASM criteria. ${ }^{17}$

To measure awake ventilatory chemoreflexes, we used a custom-made fully computerised system based on Duffin's modified rebreathing method. ${ }^{18}$ The $10 \mathrm{~min}$ full testing protocol has been described in the online supplement and elsewhere. ${ }^{18}{ }^{19}$ Two key outcome parameters, ventilatory recruitment threshold of $\mathrm{CO}_{2}$ (VRT) and hypercapnic ventilatory response slope (HCVR), a measure of chemosensitivity, are quantified using analysing software. All results were cross checked by two researchers who were blinded to the treatment order.

Plasma morphine, morphine-3-glucuronide (M3G) and morphine-6-glucuronide (M6G) concentrations were quantified by liquid chromatography mass spectroscopy at the laboratory of the Discipline of Pharmacology, The University of Adelaide, South Australia. Genotyping analysis of the ABCB1 and OPRM1 genes was also performed at this location. Genotyping analysis of the HTR3B gene was performed by the Beijing Genomics Institute, Shenzhen, Guangdong, China. The three candidate genes (OPRM1 (rs1799971, $118 \mathrm{~A}>\mathrm{G}$ ), ABCB1 (rs1045642, $3435 \mathrm{C}>\mathrm{T}$ ) and HTR3B (rs7103572 C>T)) were identified by recent studies that show different responses to opioids according to different polymorphisms. ${ }^{20-22} \mathrm{~A}$ further description of the methodology can be found in online supplement and Barrett et $a l^{23}$

\section{Outcome measures}

The primary outcome measure was the sleep time with oxygen saturation below 90\% (T90). Secondary outcomes included standard polysomnography measures during sleep, including $\mathrm{AHI}, \mathrm{SpO}_{2}$ nadir, average $\mathrm{SpO}_{2}$, oxygen desaturation index (ODI) and arousal index (ArI). Effect modifiers to be examined included awake ventilatory chemoreflex measures, plasma morphine, M3G and M6G concentrations, OPRM1, ABCB1 and HTR3B genotype.

\section{Statistical analysis}

Statistical analysis was performed in SAS version 9.4 (SAS Institute, Cary, North Carolina, USA). Analyses were planned to 
test a mixed model to determine whether sleep apnoea severity changes the effect of morphine on primary and secondary outcomes by classifying patients as having mild or moderate to severe OSA (AHI above or below 20). A test of group*drug was used to test for effect modification using an $\alpha$ of 0.05 for significance. Linear regression was used to test whether the placebo-adjusted effect of morphine on the primary outcome was associated with the following: plasma morphine concentrations (and genetic markers for morphine CNS efflux transport), awake chemoreflex parameters (HCVR/VRT), minute ventilation $\left(\mathrm{V}_{\mathrm{E}}\right)$, age and BMI. Bivariate correlations were tested by Pearson's correlation coefficient. To detect a $7 \mathrm{~min}$ difference in T90 between morphine and placebo based on a $15.4 \mathrm{~min}$ SD of the difference between morphine and placebo, observed in our pilot study with $90 \%$ power and a type one error rate of $5 \%$, 53 participants were required. ${ }^{14}$ Initially, we planned to recruit 55 patients allowing a dropout of 2 patients. Before the closing of recruitment, we considered that an extra five patients should be added, allowing for $10 \%$ of poor quality/unusable data. Therefore, a total of 60 patients were recruited. The protocol and analysis plan was pre-registered on the Australian and New Zealand Clinical Trial Registry (ACTRN12613000858796).

\section{RESULTS}

Between 21 August 2013 and 3 March 2015, 68 patients were screened for inclusion into the study (figure 1). One patient was excluded during screening due to taking lamotrigine for bipolar disorder. After consenting to participate, five patients withdrew consent for personal reasons and another could not be contacted. Sixty-one patients were randomised and completed at least one study visit, with one patient withdrawing after the placebo visit due to personal reasons. Thus, 60 patients completed the study protocol.

Baseline demographics and clinical characteristics are described in table 1 . The mean age of the 60 male patients was 44.4 years (SD 11.7) and mean BMI was in the overweight category $\left(29.3 \mathrm{~kg} / \mathrm{m}^{2}\right)$. Three patients were of Asian ethnicity, the remainder were Caucasian. The mean Epworth Sleepiness Scale score was 8.3 (SD 4.3) and mean blood pressure was within

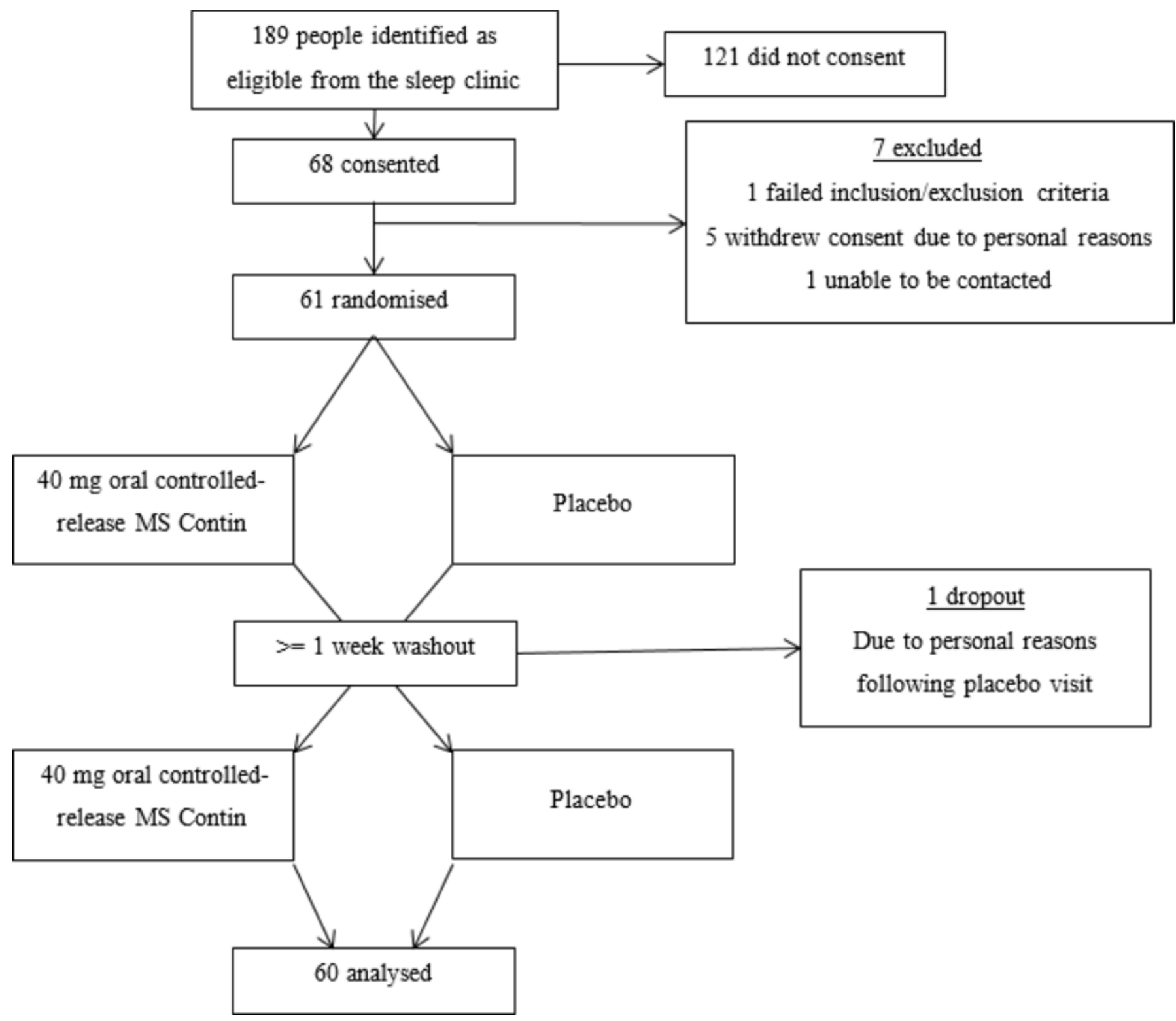

Figure 1 Study flow diagram. 
Table 1 Baseline characteristics and sleep respiratory parameters measured at placebo

\begin{tabular}{llll}
\hline & $\begin{array}{l}\text { Overall mean } \\
\text { (SD) }\end{array}$ & $\begin{array}{l}\text { Mild group } \\
\text { mean (SD) }\end{array}$ & $\begin{array}{l}\text { Severe group } \\
\text { mean (SD) }\end{array}$ \\
\hline $\mathbf{n}$ & 60 & 45 & 15 \\
\hline Age, years & $44.4(11.7)$ & $42.8(11.3)$ & $49.2(12.0)$ \\
\hline BMI, kg/m & $29.3(3.9)$ & $28.5(3.5)$ & $32.0(4.1)$ \\
\hline $\begin{array}{l}\text { Epworth Sleepiness Scale } \\
\text { (ESS), n/24 }\end{array}$ & $8.3(4.3)$ & $8.0(4.4)$ & $9.2(4.1)$ \\
\hline Neck circumference, cm & $41.2(3.4)$ & $40.2(3.0)$ & $43.9(3.3)$ \\
\hline $\begin{array}{l}\text { Systolic blood pressure, } \\
\text { mmHg }\end{array}$ & $125.8(11.1)$ & $124.9(11.6)$ & $128.6(9.4)$ \\
\hline $\begin{array}{l}\text { Diastolic blood pressure, } \\
\text { mmHg }\end{array}$ & $81.6(8.6)$ & $80.9(9.0)$ & $83.7(7.5)$ \\
\hline T90, min & $4.0(9.6)$ & $1.0(2.6)$ & $13.3(15.6)$ \\
\hline T95, min & $92.1(89.0)$ & $78.7(89.0)$ & $132.2(78.4)$ \\
\hline SpO, nadir, \% & $87.2(5.7)$ & $88.6(4.9)$ & $83.2(6.1)$ \\
\hline AHI, events/hour & $19.1(17.7)$ & $10.5(4.9)$ & $44.9(17.2)$ \\
\hline AHI (REM), events/hour & $20.3(23.4)$ & $11.8(10.5)$ & $45.6(32.2)$ \\
\hline AHI (NREM), events/hour & $18.9(17.7)$ & $10.3(5.7)$ & $44.7(16.6)$ \\
\hline ODI, events/hour & $14.3(16.0)$ & $7.0(5.0)$ & $36.0(18.2)$ \\
\hline Arl, events/hour & $25.5(13.9)$ & $20.3(7.1)$ & $41(17.7)$ \\
\hline Medications & $\mathbf{n}(\%)$ & $\mathbf{n}(\%)$ & $\mathbf{n}(\%)$ \\
\hline Antidepressant (SSRI) & $7(12)$ & $5(11)$ & $2(13)$ \\
\hline Antihypertensive & $5(8)$ & $3(7)$ & $2(13)$ \\
\hline Antihypercholesterolaemic & $6(10)$ & $4(9)$ & $2(13)$ \\
\hline Bong & & & \\
\hline
\end{tabular}

Biometrics measured at the screening visit. Polysomnography variables measured during the placebo night. Medications subjectively reported via questionnaire during screening visit. Mild group: AHI at placebo visit $<20$ events/hour; severe group: AHI at placebo visit $\geq 20$ events/hour.

AHI, apnoea-hypopnoea index; Arl, arousal index; BMI, bodymass index; N1, stage N1 sleep; N2, stage N2 sleep; NREM, non-rapid eye movement sleep; ODI, oxygen desaturation index; REM, rapid eye movement sleep; $\mathrm{SE}_{\text {, }}$ sleep efficiency; SOL, sleep onset latency; $\mathrm{SpO}_{2}$ nadir, oxygen saturation nadir; SWS, slow wave sleep; TST, total sleep time; WASO, wake after sleep onset.

the normal range. Despite our aim to have an equal number of patients distributed in the mild and severe groups, measured on the placebo night 45 patients had $\mathrm{AHI}<20$ events/hour (mild group), and 15 patients had $\mathrm{AHI} \geq 20$ events/hour (severe group) (table 1). The OPRM1 G variant allele was found in $16 / 60$ patients $(16 / 60 \mathrm{~A} / \mathrm{G} ; 0 / 60 \mathrm{G} / \mathrm{G} ; 44 / 60 \mathrm{~A} / \mathrm{A})$, the ABCB1 T variant allele in $36 / 60$ patients $(24 \mathrm{C} / \mathrm{C}$; $19 \mathrm{C} / \mathrm{T} ; 17 \mathrm{~T} / \mathrm{T})$, and the HTR3B $\mathrm{T}$ variant allele in 24 patients $(36 \mathrm{C} / \mathrm{C} ; 23 \mathrm{C} / \mathrm{T} ; 1 \mathrm{~T} / \mathrm{T})$. All were in Hardy-Weinberg equilibrium.

The primary outcome, T90, was not significantly altered by morphine, adjusting for age and BMI did not affect the result (table 2 and figure 2). Most patients had a relatively small change in T90 following morphine: $12 / 60$ patients had $0 \%$ change; $13 / 60$ patients had a small $(<5 \%$ total sleep time (TST)) improvement; $30 / 60$ patients had minor $(<5 \%$ TST) worsening. However there were a small number of patients who had more substantial changes: $3 / 60$ had improvements in T90 $>5 \%$ of TST $(15.7 \%, 8.4 \%$ and $6.1 \%)$ and $2 / 60$ patients had worsening in T90 > 5\% TST $(20.5 \%-20.9 \%$ TST).

Secondary outcomes revealed an overall mild respiratory depressant effect of morphine with sleep time below 95\% oxygen saturation (T95) increased by $41.2 \mathrm{~min}$, a reduced $\mathrm{SpO}_{2}$ nadir by $1.3 \%$, a reduced mean $\mathrm{SpO}_{2}$ by $0.5 \%$, and an increased mean transcutaneous $\mathrm{CO}_{2}$ pressure $\left(\mathrm{PtcCO}_{2}\right.$; SenTec, Switzerland) by
$2.1 \mathrm{mmHg}$ (table 2). There were no significant differences in AHI, ODI, central apnoea index (CAI) or mean apnoea length between morphine and placebo (table 2). Following morphine, 32/60 patients had a reduction in AHI (range 0.2-30.7 events/ hour), one patient had no change, and 27/60 patients had an increase in their AHI (range 0.2-40.9 events/hour). Patients spent significantly more time sleeping in the supine position with morphine (an increase of $8.6 \%$ in TST compared with placebo). However, the increase in supine sleep did not correlate with the worsening of $\mathrm{T} 95, \mathrm{SpO}_{2}$ nadir or $\mathrm{PtcCO}_{2}$ (all $\mathrm{P}$ values $>0.05$ ). OSA severity (AHI $\geq 20$ events/hour on the placebo arm) did not modify the effect of morphine therapy on any of the sleep respiratory outcomes (all interaction P values $>0.05$ ).

Morphine depressed chemosensitivity with VRT increasing by $1.6 \mathrm{mmHg}$ (Table E1) and HCVR reduced by $0.4 \mathrm{~L} / \mathrm{min} / \mathrm{mmHg}$. Baseline (on placebo) HCVR positively correlated with AHI (on placebo) $(r=0.32, P=0.01)$. In addition, the degree of HCVR reduction was positively associated with a reduction in AHI $(r=0.26, P=0.046)$. In the severe OSA group, the higher baseline (placebo) VRT correlated with an improvement in AHI, T90 (\% TST), ODI, and arousal index after morphine use (figure 3). In contrast, we did not find such a phenotyping effect in the mild OSA group with AHI $<20$ (online supplementary figure E1).

Mean plasma morphine concentration at 21:30 (3.5 hours post dose) was $7 \cdot 0$ (SD 2.6) $\mathrm{ng} / \mathrm{mL}$ and at 07:00 was 1.8 (SD 0.7) ng/mL (Table E2). Plasma morphine, M3G and M6G metabolite concentration were not associated with morphine-induced change in any key respiratory parameter in table 2 .

The OPRM1 genotype subgroup (A/G vs A/A) significantly modified morphine's effect on T90 (interaction $\mathrm{P}=0.014$ ). In 44 patients with an A/A OPRM1 genotype, T90 (\% TST) increased by $1.2 \%(\mathrm{P}=0.10)$, whereas in 16 patients with an $\mathrm{A} / \mathrm{G}$ OPRM1 genotype, T90 (\% TST) decreased by $-1.7 \%(\mathrm{P}=0.09)$. In the 44 subjects with an OPRM1 A/A genotype, HCVR was not altered by morphine $(-0.15$ (95\% CI -0.39 to 0.09$) \mathrm{L} / \mathrm{min} /$ $\mathrm{mmHg} ; \mathrm{P}=0.24)$, whereas in the $\mathrm{A} / \mathrm{G}$ subjects $(\mathrm{n}=16)$, morphine significantly reduced HCVR by $0.90(95 \% \mathrm{CI}-1.46$ to -0.33$)$ $\mathrm{L} / \mathrm{min} / \mathrm{mmHg}(\mathrm{P}=0.0041)$.

Given the links between morphine-induced change in T90 with OPRM1 genotype and baseline VRT, we also evaluated the characteristics of the five patients with the largest improvement and worsening of T90 following morphine (table 3). This showed that four of five patients who had the greatest improvements had the OPRM1 A/G genotype (only in 16/60 patients), and baseline (placebo) VRT was higher in these five patients compared with those who had the largest increases in overnight hypoxaemia. Those who had the greatest improvements were older (table 3 ).

Three of the 60 patients studied had mild adverse events which are documented in the online supplement.

\section{DISCUSSION}

This study showed that $40 \mathrm{mg}$ controlled-release oral morphine did not increase the time spent below $\mathrm{SpO}_{2}<90 \%$ during sleep (primary study outcome) or sleep apnoea frequency (secondary outcome) in individuals with OSA. However, there was large inter-individual variability. Small sub-clinical effects such as increased $\mathrm{PtcCO}_{2}$, T95 and decreased mean $\mathrm{SpO}_{2}$ were observed in some subjects. There was no relationship between the change in breathing during sleep and plasma morphine concentration. Using awake ventilatory response testing, we found that in patients with moderate to severe OSA (AHI>20 events/hour), a lower VRT correlated with worsening OSA outcomes with morphine. The study findings also support the concept that an 
Table 2 The effects of morphine on breathing during sleep

\begin{tabular}{|c|c|c|c|c|}
\hline & Placebo mean (SD) & Morphine mean (SD) & $\begin{array}{l}\text { Adjusted delta } \\
\text { mean }(95 \% \mathrm{CI})\end{array}$ & $\begin{array}{l}\text { Treatment effect } \\
\text { (P) }\end{array}$ \\
\hline T90, $\min$ & $4.0(9.6)$ & $6.2(17.8)$ & $2.1(-2.2$ to 6.4$)$ & 0.33 \\
\hline T90, \%TST & $1.2(3.1)$ & $1.6(4.6)$ & $0.4(-0.7$ to 1.6$)$ & 0.46 \\
\hline T95, $\min$ & $92.1(89.0)$ & $133.4(119.1)$ & $41.2(16.2$ to 66.3$) * *$ & 0.002 \\
\hline $\mathrm{SpO}_{2}$ nadir, $\%$ & $87.2(5.7)$ & $86(5.7)$ & $-1.3(-2.6 \text { to }-0.1)^{*}$ & 0.036 \\
\hline Mean PtcCO ${ }_{2}, \mathrm{mmHg}$ & $43.7(4.1)$ & $45.9(4.1)$ & $2.1(1.1 \text { to } 3.1)^{* * *}$ & $<0.001$ \\
\hline Mean $\mathrm{SpO}_{2}, \%$ & $95.4(1.3)$ & $94.9(1.5)$ & $-0.5(-0.7 \text { to }-0.2)^{* * *}$ & $<0.001$ \\
\hline ODI, /hour & $14.3(16)$ & $15.6(15.6)$ & $1.4(-1.6$ to 4.3$)$ & 0.36 \\
\hline AHI, /hour & $19.1(17.7)$ & $20.7(19.1)$ & $1.6(-1.9$ to 5$)$ & 0.36 \\
\hline OAI, /hour & $4.7(7.2)$ & $7.1(11.3)$ & $2.4(0.3 \text { to } 4.5)^{*}$ & 0.026 \\
\hline HI, /hour & $12.7(11.9)$ & $10.9(9.4)$ & $-1.7(-3.9$ to 0.4$)$ & 0.12 \\
\hline CAI, /hour & $0.8(1.3)$ & $1.2(2.0)$ & $0.4(-0.03$ to 0.9$)$ & 0.068 \\
\hline MAI, /hour & $1.0(2.3)$ & $1.5(2.9)$ & $0.4(-0.1$ to 1.0$)$ & 0.096 \\
\hline Arl, events/hour & $25.5(13.9)$ & $27.1(14.8)$ & $1.7(-1.9$ to 5.2$)$ & 0.35 \\
\hline Hypopnoea to apnoea ratio & $0.71(0.2)$ & $0.64(0.24)$ & $-0.07(-0.12 \text { to }-0.01)^{*}$ & 0.015 \\
\hline Mean apnoea length, s & $20.3(5.8)$ & $20.6(6.9)$ & $-0.2(-2.2$ to 1.7$)$ & 0.17 \\
\hline Supine proportion, $\%$ & $32.3(28.6)$ & $40.9(30.4)$ & $8.6(3.0 \text { to } 14.2)^{* *}$ & 0.003 \\
\hline
\end{tabular}

Bold texts indicate $p<0.05$.

$\mathrm{n}=60$. Hypopnoea to apnoea ratio = percentage of $\mathrm{AHI}$ made up of hypopnoeas, where $1=100 \%$ hypopnoeas and $0=100 \%$ apnoeas. Morphine effect tested by mixed model of repeated measures controlling for treatment order, age, BMI if appropriate. For T90 min and T90 \% TST, difference between morphine and placebo, with 95\% confidence limits, and P-value were adjusted for age, BMI, baseline T90, baseline SpO2 and proportion of sleep time spent in supine position. Supine proportion = percentage of TST spent supine.

AHI , apnoea-hypopnoea index; Arl, arousal index; BMI, body mass index; CAl, central apnoea index; HI, hypopnoea index; MAI, mixed apnoea index; OAI, obstructive apnoea index; $\mathrm{ODI}$, oxygen desaturation index; $\mathrm{PtcCO}_{2}$, pressure of transcutaneous carbon dioxide; $\mathrm{SpO}_{2}$ nadir, oxygen saturation nadir; T90 min, sleep time spent below $90 \%$ oxygen saturation; \%TST, percentage of total sleep time; T95, sleep time spent below 95\% oxygen saturation.

individual's OSA response to morphine is related to the A118G OPRM1 polymorphism.

In Pain Medicine and Anesthesiology guidelines and reviews, opioids have been implicated as worsening sleep-disordered breathing in people with OSA. ${ }^{11} 12$ However, this conclusion has been based on indirect evidence, rather than controlled clinical trials. ${ }^{10} 11$ 24-26 Existing studies and reports were confounded by being in the perioperative setting, using a higher morphine equivalent dose, concomitant use of anaesthetic agents, other sedative therapeutic drugs and/or oxygen, or involving multiple diseases (other than OSA) and surgical procedures related to pain and sleep disturbance. Therefore, it has been difficult to isolate the effect of opioids per se on OSA.

A recent Cochrane review has identified only one small controlled study that specifically evaluated the effect of opioids on OSA. ${ }^{1327}$ Within a double-blind, placebo-controlled trial, 10 patients with moderate OSA received an intravenous infusion of remifentanil $(0.075 \mathrm{mg} / \mathrm{kg}$ lean body mass) and nine different patients with OSA received a saline infusion. ${ }^{13}$ Compared with baseline, intravenous remifentanil increased mean T90 by $80 \mathrm{~min}$ and reduced $\mathrm{SpO}_{2}$ nadir by $9 \%$ awake and $7 \%$ asleep. ${ }^{13}$ In contrast, we observed no such effect either awake or asleep during our study. Interestingly, remifentanil significantly reduced OSA and increased central sleep apnoea, which led to no change in overall AHI. We did not observe any change in central or obstructive events. The difference in findings may relate to differences in drug, drug route and study design. Our oral dose of $40 \mathrm{mg}$ controlled-release morphine reflects a common dose used for pain management and is comparable to $\sim 13 \mathrm{mg}$ of intravenous morphine over a 12 hour period.

Morphine caused mild respiratory depression in the current study as reflected by the $\mathrm{T} 95, \mathrm{SpO}_{2}$ nadir and $\mathrm{PtcCO}_{2}$ during sleep findings. Recent findings indicate that increased overnight PtcCO may be an important contributor to daytime sleepiness. ${ }^{28^{2}}$ Thus, while the increase in $\mathrm{PtcCO}_{2}$ in the current study was small, morphine may contribute to daytime sleepiness via overnight $\mathrm{CO}_{2}$ retention. Increases in $\mathrm{CO}_{2}$ and reductions in oxygenation may be a primary drug effect on respiratory motor control or a secondary drug effect due to increased supine sleep in the morphine arm because of reduced motor activity with opioids. However, there was wide inter-individual variability in these parameters and the effects on breathing during sleep. Three key factors that may influence individual variation in morphine response, plasma morphine concentrations after dosing, awake chemoreflexes and genetic polymorphism, were examined in the current study. In contrast to our pilot study in which there was a robust paradoxical relationship between plasma morphine and improvement in T90, ${ }^{14}$ we found no relationship in this present trial between plasma morphine and T90. There was an eightfold range in plasma morphine and in both studies blood was sampled 4-5 hours after dosing, corresponding to the time for peak concentration.

In addition, morphine reduced awake chemosensitivity (HCVR) and increased the $\mathrm{CO}_{2}$ response threshold (VRT). Severe chemoreflex depression can be detrimental in certain patients with OSA and lead to worse blood gas disturbances. ${ }^{29}$ However, mild reductions in chemosensitivity paradoxically reduce OSA severity in the one third of patients with OSA who have heightened sensitivity to $\mathrm{CO}_{2}$, which contributes to their OSA pathogenesis. ${ }^{29}$ Indeed, interventions that reduce chemoreflex sensitivity (or high loop gain) such as $\mathrm{O}_{2}$ therapy and acetazolamide also reduce OSA severity. ${ }^{30}$ These current findings are also consistent with our pilot study using $30 \mathrm{mg}$ slow release morphine in which there was no consistent overall drug effect 


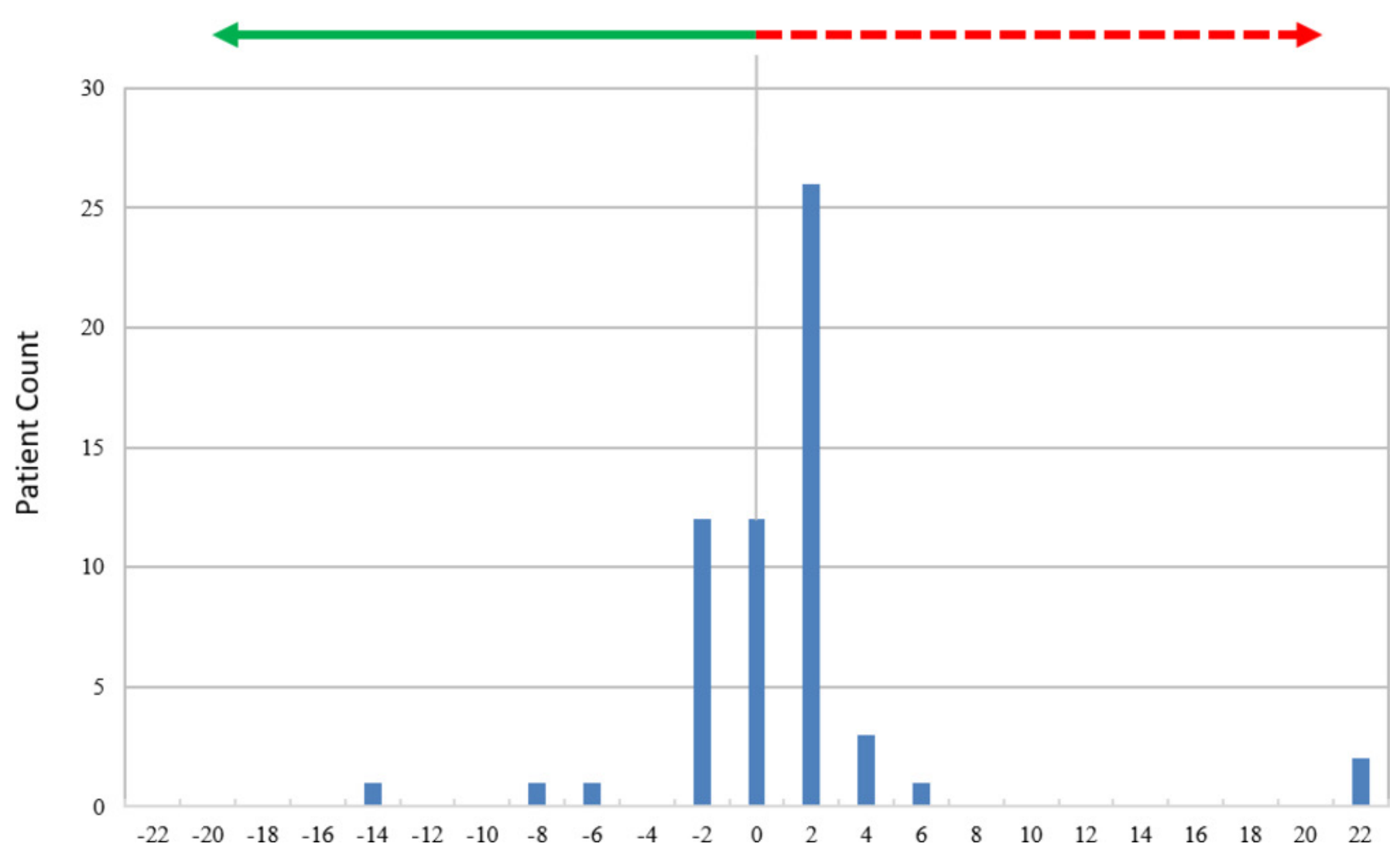

Figure 2 Histogram of change in oxygen saturation < 90\% (T90) (\% of total sleep time (TST)). Positive x-axis values (red dashed line) correspond to worsening of T90 following morphine. Negative x-axis values (solid green line) correspond to improvement in T90 following morphine.

but rather marked inter-individual variability was observed which was related to changes in chemosensitivity. ${ }^{14}$ In the present study, baseline OSA severity was associated with higher $\mathrm{HCVR} /$ chemosensitivity/loop gain, and morphine related reductions in the HCVR/loop gain were associated with a decrease in OSA severity (AHI). This is consistent with our study hypothesis. We speculate that the counter balance between morphine's OSA improving effect and direct CNS depressant effect resulted in the overall respiratory outcome of morphine on OSA. This counter balance effect may also explain the large variability in individual OSA response to morphine, and a lack of dose response respiratory depression in patients with OSA.

Prior to the study, we also hypothesised that certain baseline chemoreflex properties in patients with OSA may phenotype/ predict which patients are getting better or worse with morphine use. In the present study, we did find that in patients with severe OSA (AHI>20), higher baseline (placebo) VRT significantly correlated with an improvement in T90 (\% TST), AHI, ODI, and arousal index after morphine use (figure 3). Thus, awake chemoreflex markers may be important in predicting the effects of morphine in OSA during sleep. This predicting/phenotyping effect is not seen in the mild OSA group (online supplementary figure E1), as their baseline chemoreflex may not be significantly altered to compensate the mild upper airway abnormalities. However, given the complexity of OSA pathogenesis and variability in OSA phenotypes, ${ }^{29}$ chemoreflex sensitivity alone may be insufficient as a predictive tool.

The A/A OPRM1 genotype was associated with a worsening in T90 while improvement occurred in patients with the $A / G$
OPRM1 phenotype. This was exemplified by the finding that four of five patients who had the greatest reductions in OSA severity with morphine were A/G (in 16/60 patients). Conversely, four of the five patients who had substantial worsening of OSA with morphine were A/A (in 44/60 patients). Part of this effect may have been mediated through a reduction in chemoreflex sensitivity, with morphine reducing HCVR in $\mathrm{A} / \mathrm{G}$ patients. Thus, the clinical phenotyping and genotyping techniques used in this study may provide novel biomarkers to help predict individual responses to morphine for future larger research studies.

\section{Methodological considerations}

This is the largest randomised clinical trial to date to examine the effects of morphine in OSA and was accompanied by novel clinical phenotyping and genotyping and measurement of plasma morphine and its metabolites. However, there are several limitations that require acknowledgement. First, only men were enrolled in this study. Compared with men, women have blunted respiratory chemosensitivity which varies across the menstrual cycle and were thus excluded. ${ }^{31}$ Research in women is a priority for future appropriately designed studies. Second, the dose of morphine used in this study is common to pain management. But dosing regimens vary in different settings and there are many different types of opioids, which may have different effects on sleep and breathing, even at equivalent analgesic doses. Indeed, the findings of the current study may not necessarily extend to higher doses of opioids when the possible beneficial effects of improving respiratory chemoreflex stability could be outweighed by central depressant 

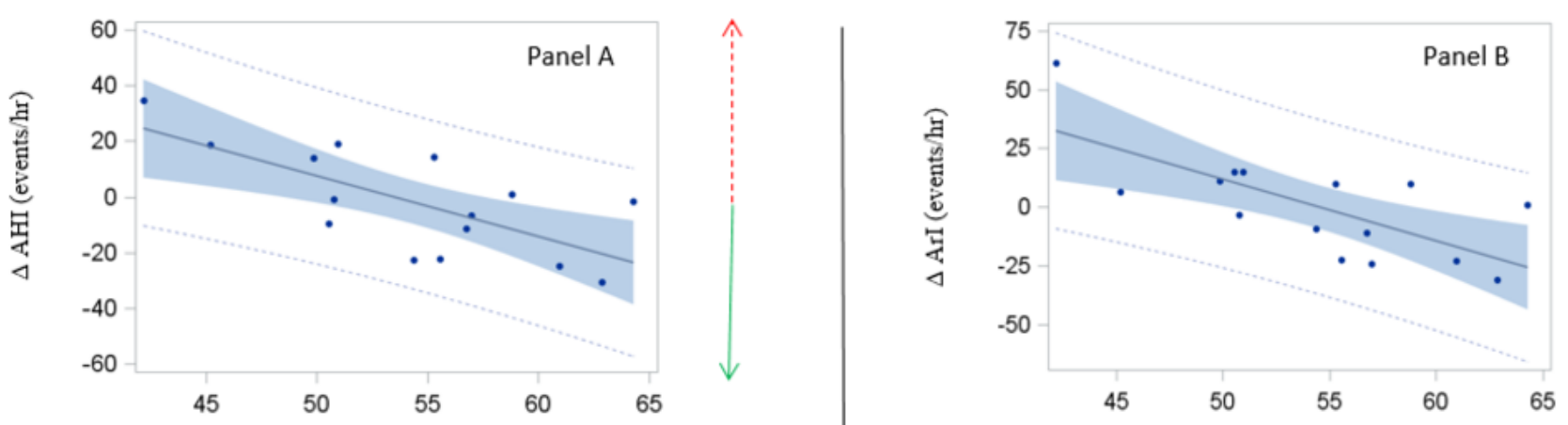

Baseline (placebo) VRT (mmHg)

Baseline (placebo) VRT (mmHg)

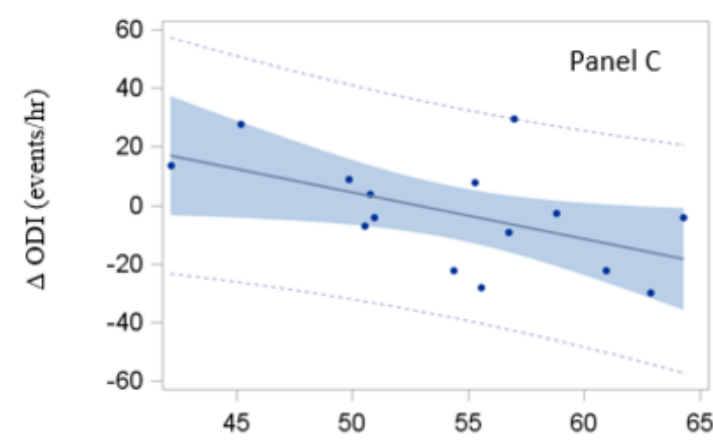

Baseline (placebo) VRT (mmHg)

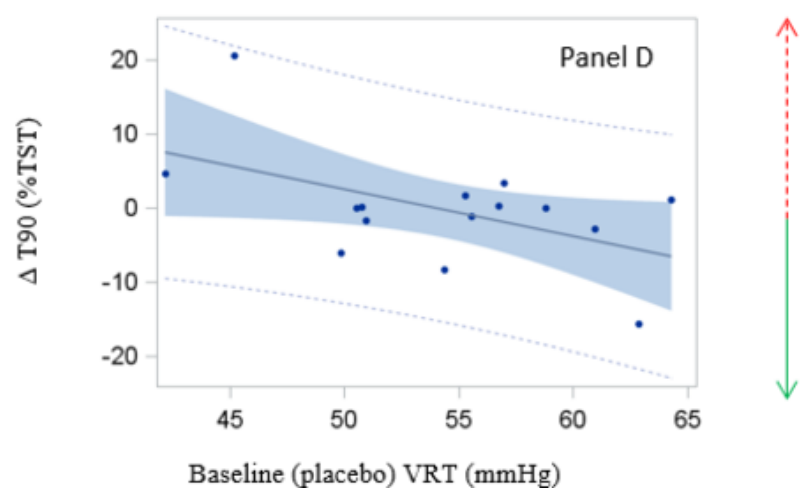

Figure 3 Correlation between placebo ventilatory recruitment threshold of $\mathrm{CO}_{2}(\mathrm{VRT})\left(\mathrm{PO}_{2}=150 \mathrm{mmHg}\right)$ and $\Delta$ respiratory polysomnographic variables in 15 patients with placebo apnoea-hypopnoea index $(\mathrm{AHI}) \geq 20$ events/hour. Dashed red arrow=worse respiration during sleep following morphine; solid green arrow=improvement. Panel A: Pearson's $r=-0.71 ; P=0.003$. Panel B: Pearson's $r=-0.71 ; P=0.003$. Panel C: Pearson's $r=-0.54$; $P=0.039$. Panel D: Pearson's $r=-0.52 ; P=0.049$.

effects. Third, we did not include patients with morbid obesity and hypoventilation due to safety concerns. In these patients, morphine could increase hypoventilation due to their reduced respiratory chemosensitivity. ${ }^{32}$ Fourth, multi-drug use and/or patients with other concurrent diseases/surgery are also beyond the scope of the current study. Fifth, we agree that the primary outcome variable

Table 3 Examination of individual characteristics of the five patients who had the greatest improvements and the five patients who had the most pronounced worsening of T90 following morphine

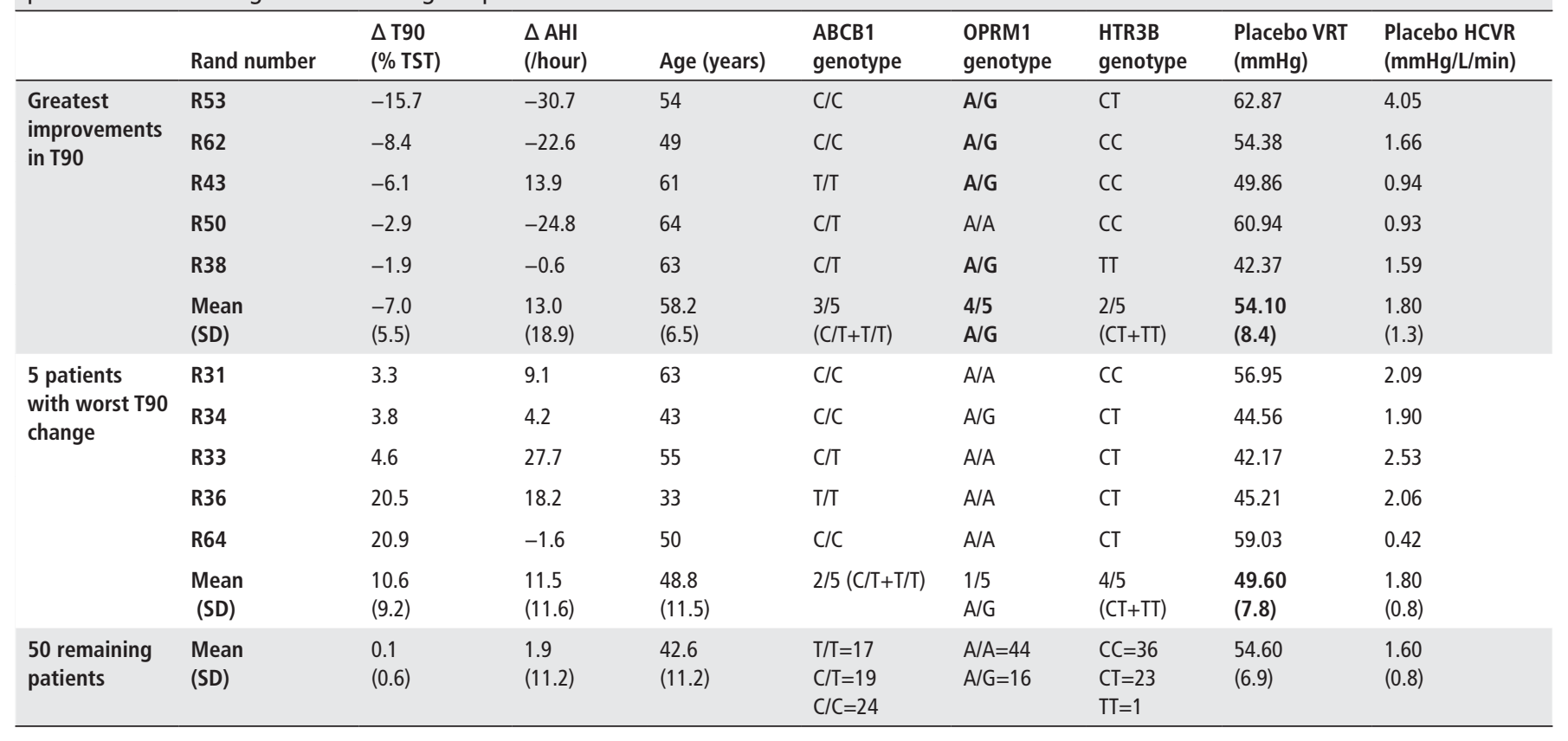


T90 min is not a perfect choice with a very skewed distribution. While most of the patients had change in T90 min of $<2 \mathrm{~min}$, a couple of patients had a change of over 1 hour. However, other PSG respiratory parameters such as $\mathrm{SpO}_{2}$ nadir, T95, T80, AHI, ODI and $\mathrm{PtcCO}_{2}$ have even greater pitfalls if selected as primary outcomes for respiratory depression. To minimise the distribution bias of T90 min, we presented T90 as percentage of total sleep time (T90\%) in analyses requiring more bell-shaped distribution. The significance of $\mathrm{P}$ values was not changed by either T90 min or T90\% (table 2). We also recognise that if total sleep time is small, T90\% could overestimate respiratory depression while T90 min could underestimate it. Finally, our acute single dose morphine results may not extend to patients with chronic morphine use in whom central sleep apnoea may be found. ${ }^{33}$ Future carefully monitored studies testing the effect of higher drug doses and chronic dosing in a broader spectrum of patients with OSA should be considered.

\section{Conclusions and clinical implications}

In conclusion, as the first major RCT in this field, we found that $40 \mathrm{mg}$ oral slow release morphine did not worsen OSA in men, challenging traditional thinking that OSA will be generally worsened with opioid use. Individual opioid response in patients with severe OSA may be related to baseline $\mathrm{CO}_{2}$ response threshold and OPRM1 genotype. Given the large variability in opioid response, we could not conclude that this dose of opioid is safe for all patients with OSA, especially in the clinical situations of morbid obesity, multi-drug use, or patients with other concurrent diseases/ surgery. Importantly, our findings in opioid response phenotype and genotype may pave the way for a precision medicine approach to develop strategies to avoid excess complications and deaths in those patients most vulnerable to opioid-related harm.

Acknowledgements We thank Dr Daniel Barratt (University of Adelaide) for the plasma concentration and genotype analyses.

Contributors Conception and design: DW, DJE and RRG. Acquisition of data: LR. Medical management: BJY and KKHW. Statistical analyses: LR, KKHW and DW. Plasma morphine, metabolites and genotyping: AAS. All authors contributed to the interpretation of data, drafting and revising of the article and final approval of article.

Funding This project was funded by Australian NHMRC Project Grant \#1043633. DW was supported by NHMRC Training Fellowship (\#571165) and NHMRC Project Grant (\#1043633). RRG was supported by NHMRC Senior Principal Research Fellowship (\#1 106974). Luke Rowsell received a postgraduate scholarship from NHMRC CRE (NeuroSleep\#1060992). DJE is supported by NHMRC Senior Research Fellowship (\#1116942).

Competing interests None declared.

Patient consent Obtained.

Ethics approval NSW Sydney Local Health District SSWAHS Ethics Committee.

Provenance and peer review Not commissioned; externally peer reviewed.

\section{REFERENCES}

1 Okie S. A flood of opioids, a rising tide of deaths. N Eng/ J Med 2010;363:1981-5.

2 Centers for Disease Control and Prevention (CDC). Unintentional poisoning deaths-United States, 1999-2004. MMWR Morb Mortal Wkly Rep 2007;56:93-6.

3 Frieden TR, Houry D. Reducing the risks of relief--the cdc opioid-prescribing guideline. N Engl J Med 2016;374:1501-4.

4 Gutstein H, Akil H. Opioid analgesics. In: Hardman JG, Limbird LE, Gilman AG, eds. Goodman and Gilman's The pharmacological basis of therapeutics. 11th edition. New York: McGraw-Hill, 2005.

5 Heinzer R, Vat S, Marques-Vidal P, et al. Prevalence of sleep-disordered breathing in the general population: the HypnoLaus study. Lancet Respir Med 2015;3:310-8.

6 Finkel KJ, Searleman AC, Tymkew H, et al. Prevalence of undiagnosed obstructive sleep apnea among adult surgical patients in an academic medical center. Sleep Med 2009;10:753-8.
7 American Society of Anesthesiologists Task Force on Perioperative Management of patients with obstructive sleep apnea. Practice guidelines for the perioperative management of patients with obstructive sleep apnea: an updated report by the American Society of Anesthesiologists Task Force on Perioperative Management of patients with obstructive sleep apnea. Anesthesiology 2014;120:268-86.

8 Chung F, Memtsoudis SG, Ramachandran SK, et al. Society of anesthesia and sleep medicine guidelines on preoperative screening and assessment of adult patients with obstructive sleep apnea. Anesth Analg 2016;123:452-73.

9 Opperer M, Cozowicz C, Bugada D, et al. Does obstructive sleep apnea influence perioperative outcome? a qualitative systematic review for the society of anesthesia and sleep medicine task force on preoperative preparation of patients with sleepdisordered breathing. Anesth Analg 2016;122:1321-34.

10 Chung F, Liao P, Elsaid $H$, et al. Factors associated with postoperative exacerbation of sleep-disordered breathing. Anesthesiology 2014;120:299-311.

11 Lam KK, Kunder S, Wong J, et al. Obstructive sleep apnea, pain, and opioids: is the riddle solved? Curr Opin Anaesthesiol 2016;29:134-40.

12 Manchikanti L, Abdi S, Atluri S, et al. American Society of Interventional Pain Physicians (ASIPP) guidelines for responsible opioid prescribing in chronic non-cancer pain: Part 2--guidance. Pain Physician 2012;15:S67-116.

13 Bernards CM, Knowlton SL, Schmidt DF, et al. Respiratory and sleep effects of remifentanil in volunteers with moderate obstructive sleep apnea. Anesthesiology 2009;110:41-9.

14 Wang D, Somogyi AA, Yee BJ, et al. The effects of a single mild dose of morphine on chemoreflexes and breathing in obstructive sleep apnea. Respir Physiol Neurobiol 2013; 185:526-32.

15 Somogyi AA, Barratt DT, Coller JK. Pharmacogenetics of opioids. Clin Pharmacol Ther 2007:81:429-44.

16 Angst MS, Lazzeroni LC, Phillips NG, et al. Aversive and reinforcing opioid effects: a pharmacogenomic twin study. Anesthesiology 2012;117:22-37.

17 Berry RB, Budhiraja R, Gottlieb DJ, et al. Rules for scoring respiratory events in sleep: update of the 2007 aasm manual for the scoring of sleep and associated events. deliberations of the sleep apnea definitions task force of the american academy of sleep medicine. J Clin Sleep Med 2012;8:597-619.

18 Duffin J, Mohan RM, Vasiliou P, et al. A model of the chemoreflex control of breathing in humans: model parameters measurement. Respir Physiol 2000;120:13-26.

19 Wang D, Marshall NS, Duffin J, et al. Phenotyping interindividual variability in obstructive sleep apnoea response to temazepam using ventilatory chemoreflexes during wakefulness. J Sleep Res 2011;20:526-32.

20 Candiotti K, Yang Z, Xue L, et al. Single-nucleotide polymorphism C3435T in the $A B C B 1$ gene is associated with opioid consumption in postoperative pain. Pain Med 2013;14:1977-84.

21 Chidambaran V, Mavi J, Esslinger $\mathrm{H}$, et al. Association of OPRM1 A118G variant with risk of morphine-induced respiratory depression following spine fusion in adolescents. Pharmacogenomics J 2015;15:255-62.

22 Currow DC, Quinn S, Ekstrom M, et al. Can variability in the effect of opioids on refractory breathlessness be explained by genetic factors? BMJ Open 2015:5:e006818.

23 Barratt DT, Coller JK, Hallinan R, et al. ABCB1 haplotype and OPRM1 118A > G genotype interaction in methadone maintenance treatment pharmacogenetics. Pharmgenomics Pers Med 2012;5:53-62.

24 Lee LA, Caplan RA, Stephens LS, et al. Postoperative opioid-induced respiratory depression: a closed claims analysis. Anesthesiology 2015;122:659-65.

25 Ramachandran SK, Haider N, Saran KA, et al. Life-threatening critical respiratory events: a retrospective study of postoperative patients found unresponsive during analgesic therapy. J Clin Anesth 2011;23:207-13.

26 Timm FP, Zaremba S, Grabitz SD, et al. Effects of opioids given to facilitate mechanica ventilation on sleep apnea after extubation in the intensive care unit. Sleep. In Press. 2018;41.

27 Mason M, Cates CJ, Smith I. Effects of opioid, hypnotic and sedating medications on sleep-disordered breathing in adults with obstructive sleep apnoea. Cochrane Database Syst Rev 2015;7:CD011090.

28 Wang $\mathrm{D}$, Thomas RJ, Yee BJ, et al. Hypercapnia is more important than hypoxia in the neuro-outcomes of sleep-disordered breathing. J Appl Physiol 2016;120:1484.

29 Eckert DJ, White DP, Jordan AS, et al. Defining phenotypic causes of obstructive sleep apnea. Identification of novel therapeutic targets. Am J Respir Crit Care Med 2013;188:996-1004.

30 Edwards BA, Sands SA, Owens RL, et al. The combination of supplemental oxygen and a hypnotic markedly improves obstructive sleep apnea in patients with a mild to moderate upper airway collapsibility. Sleep 2016;39:1973-83.

31 Jensen $D$, Wolfe LA, O'Donnell DE, et al. Chemoreflex control of breathing during wakefulness in healthy men and women. J App/ Physiol 2005;98:822-8.

32 Manuel AR, Hart N, Stradling JR. Correlates of obesity-related chronic ventilatory failure. BMJ Open Respir Res 2016;3:e000110.

33 Wang D, Teichtahl H, Drummer 0 , et al. Central sleep apnea in stable methadone maintenance treatment patients. Chest 2005;128:1348-56. 